\title{
Excited-State Proton Transfer of 1-Naphthol in Micelles
}

\author{
Debabrata Mandal, Samir Kumar Pal, and Kankan Bhattacharyya* \\ Physical Chemistry Department, Indian Association for the Cultivation of Science, Jadavpur, \\ Calcutta 700 032, India
}

Received: June 4, 1998; In Final Form: August 24, 1998

\begin{abstract}
The fast deprotonation of 1-naphthol, which occurs in $35 \mathrm{ps}$ in aqueous solution, is studied in neutral (triton X 100, reduced, TX-100R), cationic (cetyl trimethylammonium bromide, CTAB), and anionic (sodium dodecyl sulfate, SDS) micelles. Drastically different effects on the proton transfer process and the relative emission intensities of the neutral form $(360 \mathrm{~nm})$ and the anion $(460 \mathrm{~nm})$ are observed in the three micelles. The intensities of the anion and the neutral emission of 1-naphthol exhibit a break around the reported critical micellar concentration (cmc) of the three micelles. Above cmc, intensity of the neutral emission is enhanced by a factor of nearly 90, 66, and 20 in $20 \mathrm{mM}$ TX-100R, $200 \mathrm{mM}$ SDS, and $96 \mathrm{mM}$ CTAB, respectively. The anion emission is enhanced for CTAB and TX-100R, while for SDS its intensity decreases, compared to water. In CTAB, the rise time of the $460 \mathrm{~nm}$ emission ( $600 \pm 100 \mathrm{ps})$ is similar to the lifetime of decay at $360 \mathrm{~nm}$. However, for TX-100R and SDS, the rise time of the anion emission (at $460 \mathrm{~nm}$ ) is found to be faster than the decay of the neutral emission (at $360 \mathrm{~nm}$ ). This indicates that in TX-100R and SDS, there is no parental relation between the normal and the anion emission and they originate from the probe, 1-naphthol molecules, at distinctly different locations. The rise times at $460 \mathrm{~nm}$ are $1.8 \pm 0.1 \mathrm{~ns}$ and $600 \pm 100 \mathrm{ps}$ for TX-100R and SDS, respectively, while the corresponding decay times at $360 \mathrm{~nm}$ are $2.5 \pm 0.1 \mathrm{~ns}$ and $1.8 \pm$ $0.1 \mathrm{~ns}$.
\end{abstract}

\section{Introduction}

The structure, equilibrium, and dynamics in confined environments and at various interfaces play a crucial role in many biological and natural processes. ${ }^{1}$ Many ultrafast processes become significantly retarded in such environments. For instance, the rate of the intramolecular charge-transfer processes of different probes are reduced by 1-2 orders of magnitude in microemulsions ${ }^{2}$ and in other organized media. ${ }^{3}$ The ultrafast excited-state intramolecular proton transfer process is markedly slowed inside the cyclodextrin cavities. ${ }^{4}$ More dramatic retardation has been observed in the case of solvation dynamics. While solvation dynamics of water molecules occurs in subpicosecond time scale in ordinary water, ${ }^{5 a}$ it is retarded by at least 3 orders of magnitude to the nanosecond time scale inside the cyclodextrin cavities, ${ }^{5 \mathrm{a}, \mathrm{b}}$ micelles, ${ }^{6}$ and microemulsions. ${ }^{7}$ The intermolecular proton transfer processes are also found to be markedly affected by cyclodextrins, ${ }^{8-11}$ lipids, ${ }^{12}$ and microemulsions. ${ }^{13}$ The excited-state double proton-transfer process ${ }^{15}$ is also largely modified in microemulsions. ${ }^{14}$ In general, the aromatic amines and phenols become more acidic in the excited state and their $\mathrm{p} K_{\mathrm{a}}$ decreases by several units in the electronically excited state compared to that in the ground state. ${ }^{16}$ As a result, if the $\mathrm{pH}$ of the medium is intermediate between the $\mathrm{p} K_{\mathrm{a}}$ in the ground and the excited states, while in the ground state the molecule remains in the neutral form, in the excited state, it readily deprotonates to produce the anion in the excited state, from which the Stokes shifted emission originates. Thus, in aqueous medium the time-resolved fluorescence of the neutral form displays a decay and the anion emission exhibits an initial rise, followed by a decay.

* Author to whom correspondence should be addressed. Fax: 91-33473-2805. E-mail: pckb @ mahendra.iacs.res.in.
Among the aromatic phenols, 1-naphthol ${ }^{17-22}$ and its derivatives $^{23}$ stand out due to their extremely fast deprotonation rate. In aqueous solution, 1-naphthol undergoes deprotonation in 35 ps, causing a 35 ps lifetime of the emission from the neutral form (at $360 \mathrm{~nm}$ ) and a $35 \mathrm{ps}$ rise time for the anion (at 460 $\mathrm{nm}){ }^{18}$ As a result of the ultrafast deprotonation, in aqueous medium, the intensity of the neutral emission of 1-naphthol is extremely low in aqueous solution and one observes almost exclusively the anion emission. In alcohol, however, the deprotonation is markedly suppressed and only the neutral emission is observed. ${ }^{19}$ Fleming et al. demonstrated that in aqueous solutions the rate of deprotonation of protonated aminopyrene increases nearly 3 times when the probe binds with cyclodextrin, while for 1-naphthol the deprotonation becomes almost 20 times slower. ${ }^{9}$ Although the effect of cyclodextrin, ${ }^{8-11}$ lipids, ${ }^{12}$ and different solvent mixtures ${ }^{17,19-24}$ on the excited state proton transfer (ESPT) processes of 1-naphthol and other compounds is well studied, there has been little study on the effect of micelles on the ultrafast deprotonation of 1-naphthol. In the present work, we will show that the binding of 1-naphthol to micelles (neutral, Triton X 100, reduced, TX-100R, cationic, cetyl trimethylammonium bromide, CTAB, and anionic, sodium dodecyl sulfate, SDS) dramatically affects the dynamics of the excited-state proton transfer (ESPT) in 1-naphthol.

\section{Experimental}

1-Naphthol (Merck, 99.9+\%) was purified by vacuum sublimation followed by recrystallization from 1:1 aqueous methanol. Triton X 100, reduced (Nacalai Tesque), CTAB, and SDS (Aldrich) were used as received. The steady-state absorption and emission spectra were recorded in a JASCO 7850 spectrophotometer and a Perkin-Elmer 44B spectrofluorimeter, respectively. For lifetime measurement, the sample was excited 

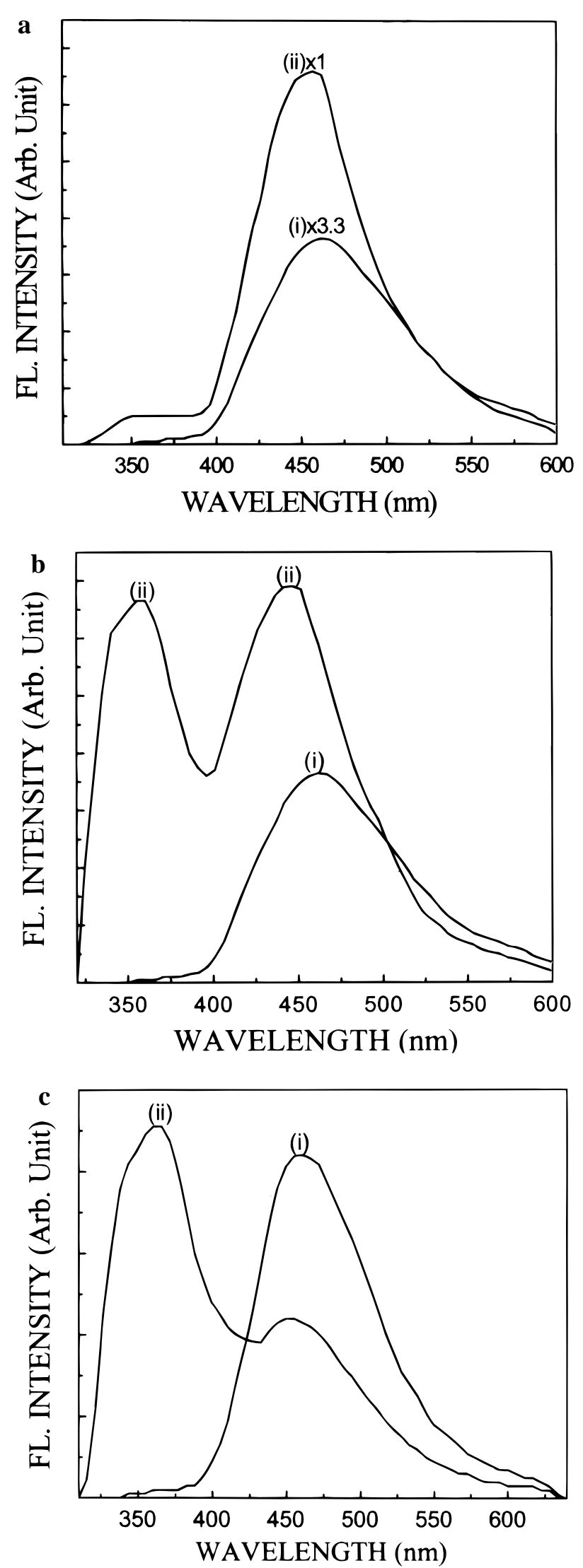

Figure 1. Emission spectra of $5 \times 10^{-5} \mathrm{M}$ 1-naphthol in (a) (i) water, (ii) $96 \mathrm{mM} \mathrm{CTAB}$; (b) (i) water, (ii) $20 \mathrm{mM} \mathrm{TX}-100 \mathrm{R}$; (c) (i) water, (ii) $200 \mathrm{mM}$ SDS.

with the second harmonic of a cavity-dumped Rhodamine 6G dye laser (Coherent 702-1) pumped by a cw mode-locked $\mathrm{Nd}$ : YAG laser (Coherent Antares 76s). The emission was detected
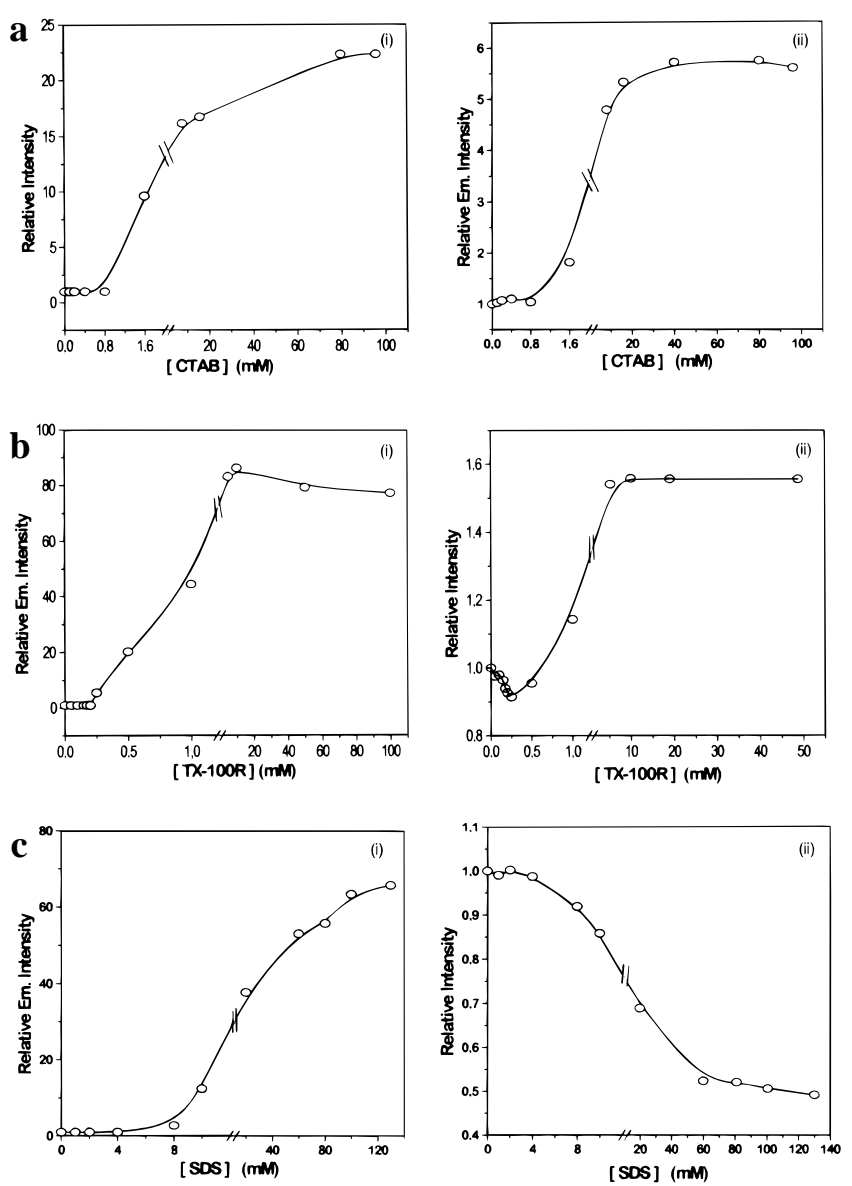

Figure 2. Plot of relative emission intensity of $5 \times 10^{-5} \mathrm{M}$ 1-naphthol against concentration of (a) CTAB at $\lambda_{\mathrm{em}}$, (i) $360 \mathrm{~nm}$, (ii) $460 \mathrm{~nm}$; (b) TX-100R at $\lambda_{\mathrm{em}}$, (i) $360 \mathrm{~nm}$, (ii) $460 \mathrm{~nm}$; (c) SDS at $\lambda_{\mathrm{em}}$, (i) $360 \mathrm{~nm}$, (ii) $460 \mathrm{~nm}$.

at magic angle polarization using a Hamamatsu MCP photomultiplier (2809U). The fluorescence decays were deconvoluted using a global lifetime analysis software (PTI).

\section{Results}

1. Steady-State Emission Spectra. Figures $1 \mathrm{a}-\mathrm{c}$, respectively, illustrate the effect of CTAB, TX-100R, and SDS on the emission spectra of 1-naphthol. In aqueous solution emission of the neutral form (1-naphthol) at $360 \mathrm{~nm}$ is very weak $\left(\phi_{\mathrm{f}}=0.002\right)$, while the intensity of the anion emission, at $460 \mathrm{~nm}$, is moderately strong $\left(\phi_{\mathrm{f}}=0.114\right) .{ }^{19}$ On addition of the surfactants, the emission intensity of the neutral and the anion of 1-naphthol, remains unchanged up to about $0.9 \mathrm{mM}$ CTAB, $0.2 \mathrm{mM}$ TX-100R, and $8 \mathrm{mM}$ SDS which are close to the reported critical micellar concentration $(\mathrm{cmc})^{25}$ of these micelles (Figure 2). Above cmc, at $96 \mathrm{mM} \mathrm{CTAB}$, the neutral $(360 \mathrm{~nm})$ and the anion emission (at $460 \mathrm{~nm}$ ) exhibit, respectively, nearly 20- and 6-fold increase in the emission intensity, compared to water (Figure 1a). For TX-100R, above $\mathrm{cmc}$, there is a very significant increase in the intensity of the neutral emission by nearly 90 times at $20 \mathrm{mM}$ TX-100R (Figure 1b), and along with this the anion emission exhibits 1.5 -fold enhancement. Unlike CTAB and TX-100R, for SDS, above cmc, the intensity of the anion emission decreases about 2 times at $[\mathrm{SDS}]=200 \mathrm{mM}$, while the intensity of the neutral emission increases markedly by about 66 times (Figures $1 \mathrm{c}$ and 2c). In all three micelles, the excitation spectra monitored at both the neutral and the anion emission peaks remain identical to the 

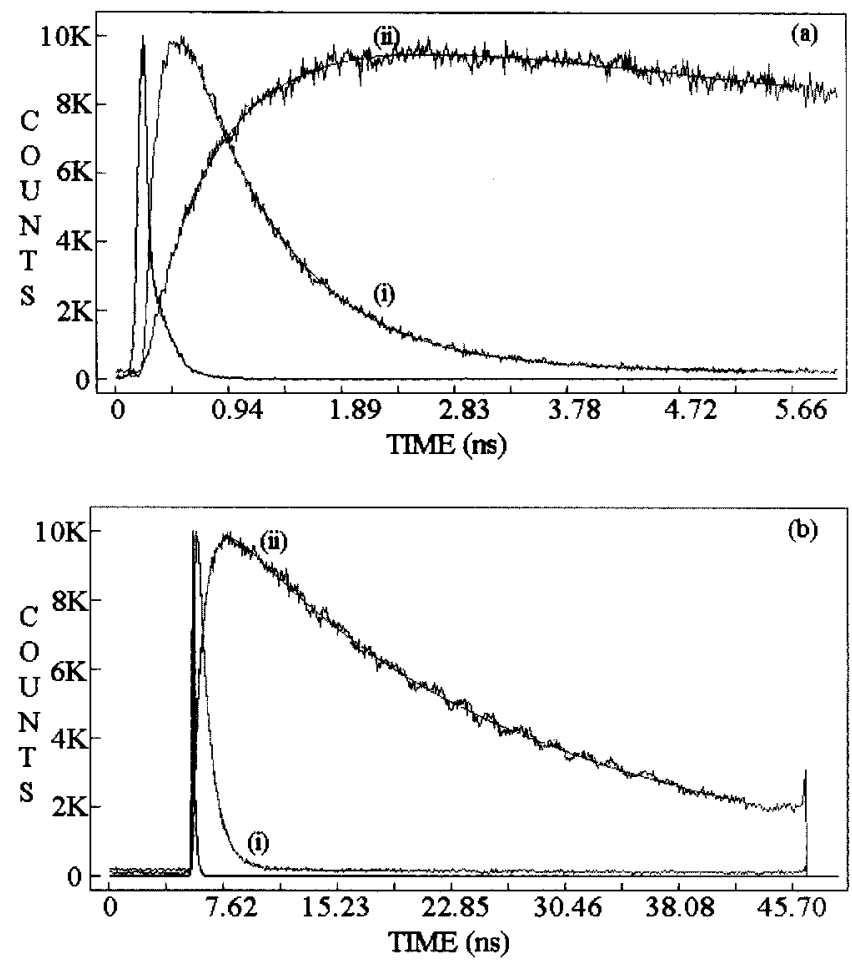

Figure 3. Fluorescence decay of $5 \times 10^{-5} \mathrm{M} 1$-naphthol in $20 \mathrm{mM}$ TX-100R, (a) resolution $=0.0244 \mathrm{~ns} / \mathrm{ch}$, (i) $\lambda_{\mathrm{em}}=360 \mathrm{~nm}$, (ii) $\lambda_{\mathrm{em}}=$ $460 \mathrm{~nm}$; (b) resolution $=0.0952 \mathrm{~ns} / \mathrm{ch}$, (i) $\lambda_{\mathrm{em}}=360 \mathrm{~nm}$, (ii) $\lambda_{\mathrm{em}}=$ $460 \mathrm{~nm}$.

absorption spectra of 1-naphthol. This rules out involvement of any impurity.

2. Time-Resolved Studies. It is difficult to extract meaningful decay times in such inhomogeneous micellar systems, as the probe may exist in drastically different locations, e.g., the bulk water, hydrocarbon core of the micelles, and the micelle-water interface. ${ }^{26}$ Obviously, the decay of the emissions are expected to be multiexponential. To minimize the contribution of the free 1-naphthol in bulk water, all the decays were recorded at a concentration much higher than the $\mathrm{cmc}$ of the surfactants, when almost all the probe 1-naphthol molecules remain bound to the micelles. Figures 3-5, respectively, depict the fluorescence decays of 1-naphthol in $20 \mathrm{mM}$ TX-100R, 96 $\mathrm{mM} \mathrm{CTAB}$, and $200 \mathrm{mM}$ SDS, in aqueous solutions, at 360 $\mathrm{nm}$ (neutral emission) and $460 \mathrm{~nm}$ (anion emission), at two different time scales.

It is readily seen that in $96 \mathrm{mM} \mathrm{CTAB}$, the lifetime of the decay of the neutral form and the rise time of the anion emission is $600 \pm 100$ ps (Figure 3a), which is nearly 20 times that in water. $9,18,19$ This shows that the deprotonation of 1-naphthol is slowed by a factor of 20 on binding to CTAB. The lifetime of the decay of the anion emission is found to be $19 \pm 0.5 \mathrm{~ns}$ which is nearly two times longer than the lifetime of the anion in water $(8 \mathrm{~ns}){ }^{19}$ Figure $3 \mathrm{~b}$, further, demonstrates that the neutral emission contains no slow component and becomes essentially complete long before the anion emission is extinguished.

For neutral TX-100R, the rise time of the anion emission of 1-naphthol $(1.9 \pm 0.1 \mathrm{~ns})$ is found to be faster than the decay of the normal emission (2.6 $\pm 0.1 \mathrm{~ns})$ (Figure 4a). The lifetime of the decay of the anion emission in $20 \mathrm{mM}$ TX $100 \mathrm{R}$ is found to be $14 \pm 0.5 \mathrm{~ns}$ (Figure 4b).

In $200 \mathrm{mM}$ SDS, also, it is observed that the rise time of the anion emission $(600 \pm 100 \mathrm{ps})$ is much faster than the decay of the normal emission $(1.8 \pm 0.2 \mathrm{~ns})$. In SDS, the anion
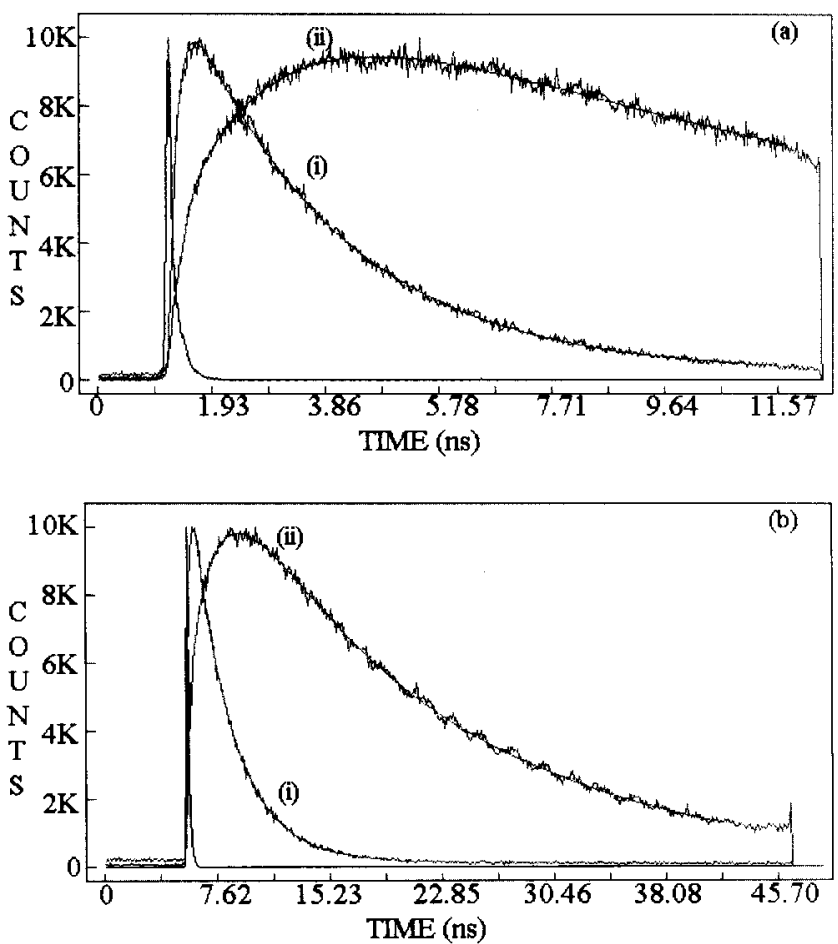

Figure 4. Fluorescence decay of $5 \times 10^{-5} \mathrm{M} 1$-naphthol in $96 \mathrm{mM}$ $\mathrm{CTAB}$, (a) resolution $=0.0118 \mathrm{~ns} / \mathrm{ch}$, (i) $\lambda_{\mathrm{em}}=360 \mathrm{~nm}$, (ii) $\lambda_{\mathrm{em}}=$ $460 \mathrm{~nm}$; (b) resolution $=0.0952 \mathrm{~ns} / \mathrm{ch}$, (i) $\lambda_{\mathrm{em}}=360 \mathrm{~nm}$, (ii) $\lambda_{\mathrm{em}}=$ $460 \mathrm{~nm}$.
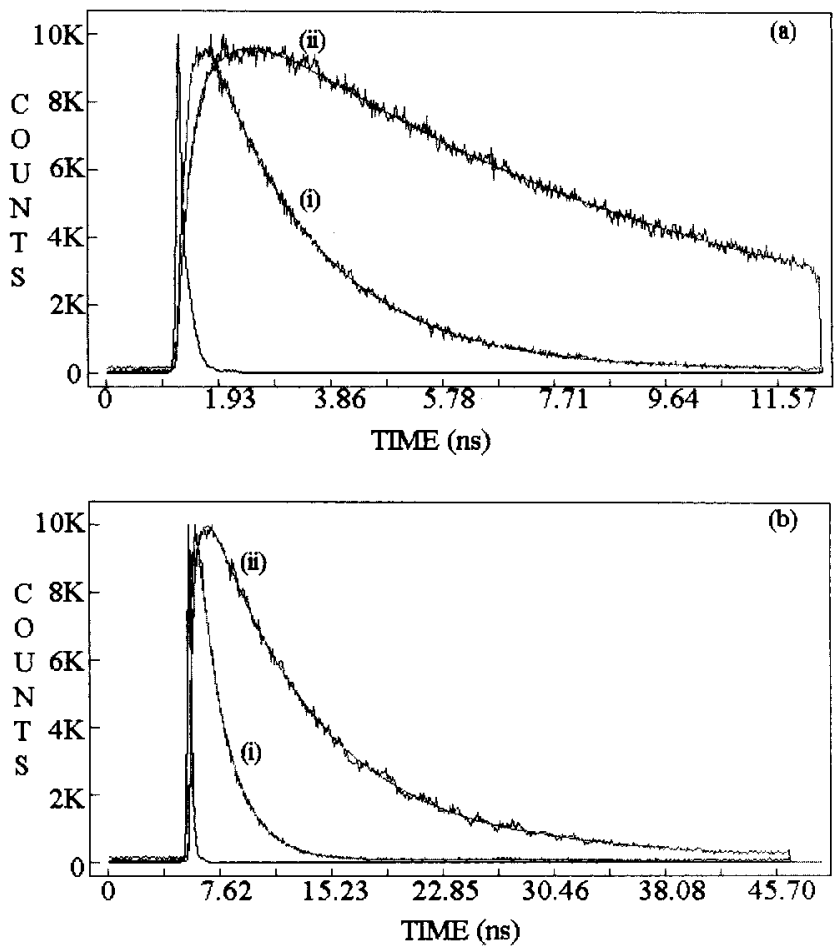

Figure 5. Fluorescence decay of $5 \times 10^{-5} \mathrm{M}$ 1-naphthol in $200 \mathrm{mM}$ SDS, (a) resolution $=0.0244 \mathrm{~ns} / \mathrm{ch}$, (i) $\lambda_{\mathrm{em}}=360 \mathrm{~nm}$, (ii) $\lambda_{\mathrm{em}}=460$ $\mathrm{nm}$; (b) resolution $=0.0952 \mathrm{~ns} / \mathrm{ch}$, (i) $\lambda_{\mathrm{em}}=360 \mathrm{~nm}$, (ii) $\lambda_{\mathrm{em}}=460$ $\mathrm{nm}$.

emission decays with a lifetime $7.8 \pm 0.2 \mathrm{~ns}$, which is pretty close to the lifetime of the decay of the anion emission, in bulk water.

It may be recalled that in mixed solvents, Robinson et al. earlier reported that at high alcohol content, the rise at $460 \mathrm{~nm}$ is faster than the decay at $360 \mathrm{~nm} .{ }^{19}$ As a control experiment, 


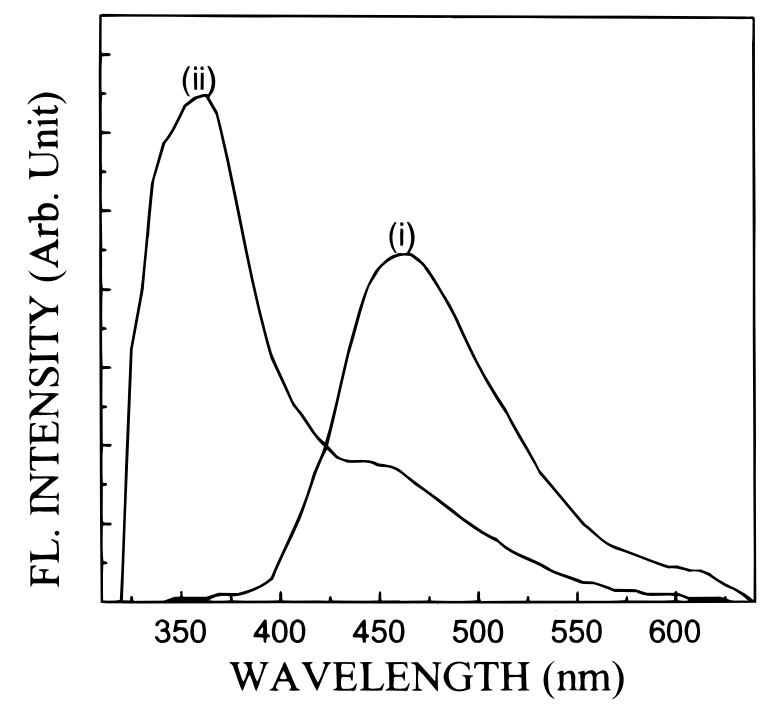

Figure 6. Emission spectra of $5 \times 10^{-5} \mathrm{M}$ 1-naphthol in (i) water and (ii) $90 \%$ methanol/water (v/v).
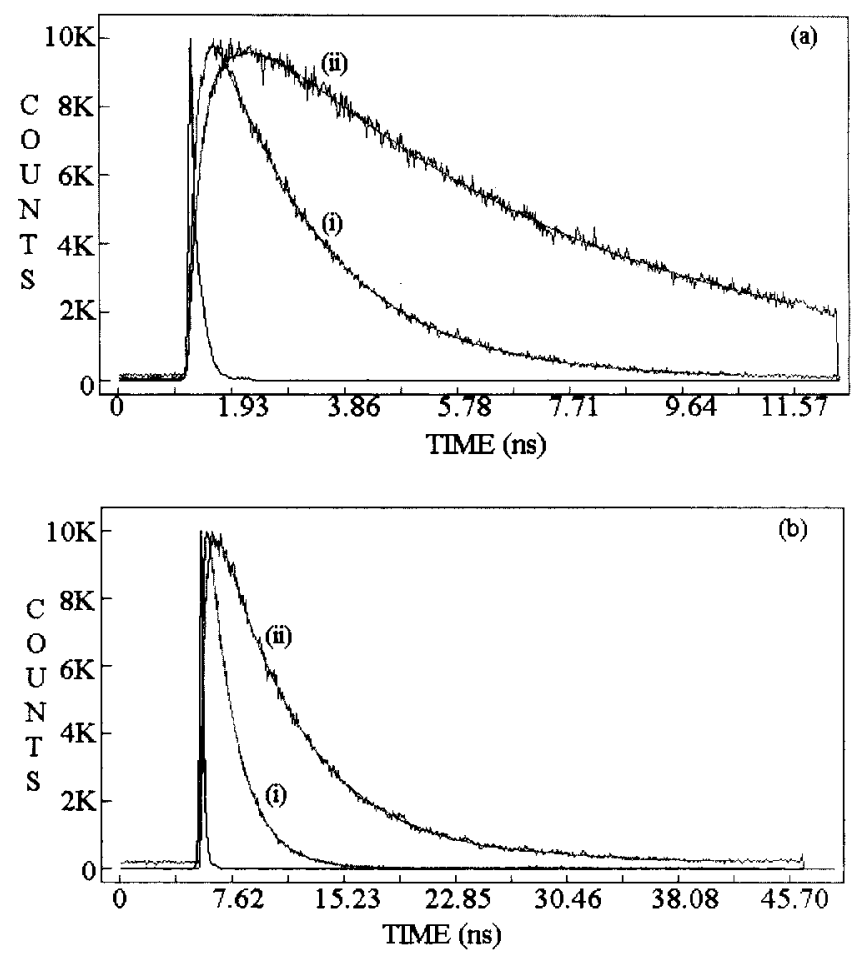

Figure 7. Fluorescence decay of $5 \times 10^{-5} \mathrm{M}$ 1-naphthol in $90 \%$ methanol/water $(\mathrm{v} / \mathrm{v})$ (a) resolution $=0.0244 \mathrm{~ns} / \mathrm{ch}$, (i) $\lambda_{\mathrm{em}}=360 \mathrm{~nm}$, (ii) $\lambda_{\mathrm{em}}=460 \mathrm{~nm}$; (b) resolution $=0.0952 \mathrm{~ns} / \mathrm{ch}$, (i) $\lambda_{\mathrm{em}}=360 \mathrm{~nm}$, (ii) $\lambda_{\mathrm{em}}=460 \mathrm{~nm}$.

we studied 1-naphthol, in $90 \%$ methanol in water (v/v). The steady-state spectrum shows the contribution of the anion emission is much less than that of the neutral emission in $90 \%$ methanol (Figure 6) and the rise time at $460 \mathrm{~nm}(600 \pm 100$ ps) is faster than the lifetime of the decay at $360 \mathrm{~nm}(1.8 \pm 0.1$ ns) (Figure 7).

\section{Discussion}

The present work demonstrates that, compared to water, in the three micelles the excited state deprotonation of 1-naphthol is significantly retarded. This results in a substantial increase in the intensity and the lifetime of the neutral emission and the rise time of the anion emission. Since the fast deprotonation of 1-naphthol is observed only in aqueous medium and is completely suppressed in even alcohols, ${ }^{19-21}$ the reduction in the deprotonation rate may be ascribed to the lower polarity of the micellar media and the lower accessibilty of the 1-naphthol molecules, encaged in the micelles, to the water molecules. As noted earlier, similar dramatic reduction in the rates of other ultrafast processes has been observed earlier in many organized media. $^{2-7}$

It is also important to note, that at long times the neutral and the anion emission do not decay with the same lifetime. This indicates that no equilibrium is established between the neutral and the anion form, in the electronically excited state when 1-naphthol binds to any of the three micelles. The failure to establish an equilibrium may be ascribed to the extreme slowness of the back proton transfer or reprotonation of the 1-naphtholate anion. This may be due to the steric hindrance posed by the hydrogen atoms at the 1 '-position of the adjacent ring of the naphthalene moiety.

The enhancement of the anion emission is, obviously, due to the reduction in the nonradiative rates of the anion inside the micelles, as is evidenced by the increase in the lifetime of the anion emission from $8 \mathrm{~ns}$ in water to nearly $19 \mathrm{~ns}$ in CTAB and $14 \mathrm{~ns}$ in TX-100R.

The difference in the behavior of the three micelles may be attributed to the surface charges, local $\mathrm{pH}$, and the structure of the three micelles. In recent years, detailed information on the structure of these micelles has been obtained using small-angle X-ray and neutron scattering and other techniques. ${ }^{27}$ These studies indicate that these micelles consist of a "dry" hydrocarbon core surrounded by a "wet" spherical shell, called the Stern layer. The thickness of the Stern layer is 6-9 $\AA$ for CTAB and SDS. However, for TX-100R, the Stern layer is much thicker $(25 \AA)$. The Stern layer contains the counterions for SDS and CTAB and a considerable amount of water. Between the Stern layer and bulk water, there is the very diffuse Gouy-Chapman (GC) layer. Evidently for CTAB and SDS, due to the thinness of the Stern layer, the probe 1-naphthol molecule remains partially exposed to the GC layer while for TX-100R the probe is almost entirely confined to the much thicker Stern layer. Obviously, the water molecules and the hydroxyl ions are the main candidates to abstract the proton from 1-naphthol. Robinson et al. earlier proposed that around four water molecules are needed to solvate a proton. ${ }^{20,21}$ As a result, the deprotonation rate depends on the accessibility of the proton of the probe photoacid to the water molecules. When the probe, 1-naphthol, is transferred to the interior of the micelles, it becomes less accessible to the bulk water molecules. Though, at first sight, one might expect that the lower accessibility toward bulk water automatically implies slower deprotonation rate, the actual situation depends on the probe used. Several groups have investigated the ESPT process of different probe molecules in different solvent mixtures. ${ }^{17,19-21}$ For protonated aminopyrene, Pines and Fleming reported that in a water-alcohol mixture the deprotonation rate actually increases with alcohol concentration up to about $65-70 \%$ and at higher alcohol concentrations the deprotonation process is retarded. ${ }^{17}$ The faster deprotonation, of protonated aminopyrene, inside the cyclodextrin cavity, may thus be attributed to a microenvironment, between pure water and 65\% alcohol in water. ${ }^{9}$ For 1-naphthol, on the other hand, Robinson et al. showed that the deprotonation rate monotonically decreases as the alcohol content increases. ${ }^{19}$ Fleming et al. reported that deprotonation of 1-naphthol is 20 times retarded inside cyclodextrin cavities. ${ }^{9}$ The reduction in the rate of ESPT, in this case, may be due to a microenvironment less polar and less protic than bulk water. The dramatic reduction in the rate of excited-state deprotonation, as manifested in the increase in the 
lifetime of the neutral emission at $360 \mathrm{~nm}$ and the rise time of the anion emission (460 nm), for CTAB, SDS, and TX-100R, may similarly arise as a result of a less polar and less protic environment with lower accessibility to bulk water. Robinson et al. ${ }^{19}$ also reported that at high alcohol content the rise time of the anion is faster than the decay of the neutral form. This indicates that in alcohol-water mixtures, the anion and the neutral form originate from different sets of molecules. The results of the present work can be understood in the light of these observations.

For CTAB, the very similar rise time at $460 \mathrm{~nm}$ and the decay time at $360 \mathrm{~nm}$ indicates all the probe 1-naphthol molecules experience more or less a similar environment and the anion is formed as a result of the decay of the neutral form. Due to its inherent positive charge, CTAB is expected to have the lowest proton concentration and highest hydroxyl ion concentration in its immediate vicinity and this should facilitate the deprotonation process.

For the neutral (TX 100R) and the anionic SDS micelles, the local hydroxyl ion concentrations around the surfactants are lower than that in the case of CTAB. This leads to a slower rate of deprotonation and, consequently, higher intensity of the neutral emission. For TX-100R and SDS, the difference in the rise time at $360 \mathrm{~nm}$ and the decay at $460 \mathrm{~nm}$ indicates broadly that there are two sets of molecules. One of them gives exclusively the neutral emission and the other produces the anion emission. It seems that both in $90 \%$ methanol and inside the TX-100R and SDS micelles, all the probe 1-naphthol molecules do not get a large enough number $(4 \pm 1)^{20,21}$ of water molecules in their immediate neigborhood to undergo the fast deprotonation. Those which get a sufficient number of water molecules undergo fast deprotonation to give exclusively the anion emission, while those which are less accessible to water molecules give predominantly the neutral emission.

For SDS, a marked decrease in the intensity of the anion emission is observed despite the fact that the lifetime and hence the nonradiative rate remains the same. The similarity in the lifetime of the anion emission in water, with and without SDS, suggests that in the presence of SDS, the anion emission originates mainly from the free probe molecules in bulk water. The decrease in the intensity of the anion emission is presumably due to the transfer of the probe 1-naphthol molecules from bulk water to the interior of the micelles. For SDS the enhanced normal emission arises from 1-naphthol molecules in the interior of the micelles where the lower local hydroxyl concentration around the anionic SDS surfactant causes significant supression of the deprotonation process and thus gives almost exclusively the neutral emission at the expense of the anion emission.

\section{Conclusions}

The present work demonstrates that the very fast deprotonation of 1-naphthol is significantly retarded inside the SDS, CTAB, and TX-100R micelles. This results in a marked increase in the intensity and lifetime of the neutral emission and the rise time of the anion emission. However, while for cationic $\mathrm{CTAB}$ the rise time of the anion emission is similar to the decay time of the neutral emission, for SDS and TX-100R the rise times are faster than the decay times of the neutral form. This is attributed to the presence of the probe molecules in different locations. This is consistent with the earlier observation that even in alcohol-water mixtures with high alcohol content the rise time of the anion emission is faster than the decay time of the neutral form. ${ }^{19}$ For the anion for CTAB and TX-100R, a large increase in the intensity as well as the lifetime is observed. For SDS, however, the intensity of the anion emission decreases while its lifetime remains very close to that of the free probe in water. This indicates that in SDS the anion emission arises mainly from the free probe molecules in bulk water.

Acknowledgment. Thanks are due to the Department of Science and Technology and Council of Scientific and Industrial Research (CSIR), Government of India, for generous research grants. D.M. and S.K.P. thank CSIR for providing fellowships.

\section{References and Notes}

(1) (a) Eisenthal, K. B. Chem. Rev. 1996, 96, 1343. (b) Zhu, S.-B.; Singh, S.; Robinson, G. W. Adv. Chem. Phys. 1994, 85, 627.

(2) (a) Cho, C. H.; Chung, M.; Lee, J.; Nguyen, T.; Singh, S ; Vedamuthu, M.; Yao, S.; Zhu, S.-B.; Robinson, G. W. J. Phys. Chem. 1995 99, 7806. (b) Datta, A.; Mandal, D.; Pal, S. K.; Bhattacharyya, K. J. Phys. Chem. B 1997, 101, 10221.

(3) Bhattacharyya, K.; Chowdhury, M. Chem. Rev. 1993, 93, 507.

(4) Douhal, A.; Fiebig, T.; Chachisvilis, M.; Zewail, A. H. J. Phys. Chem. A 1998, 102, 1657.

(5) (a) Vajda, S.; Jimenez, R.; Rosenthal, S. J.; Fidler, V.; Fleming, G. R.; Castener, E. J., Jr. J. Chem. Soc., Faraday Trans. 1995, 91, 867. (b) Nandi, N.; Bagchi, B. J. Phys. Chem. 1996, 100, 13955.

(6) Sarkar, N.; Datta, A.; Das, S.; Bhattacharyya, K. J. Phys. Chem. 1996, $100,15423$.

(7) (a) Lundgren, J. S.; Heitz, M. P.; Bright, F. V. Anal. Chem. 1995 , 67, 3775. (b) Das, S.; Datta, A.; Bhattacharyya, K. J. Phys. Chem. A 1997, 101, 3299. (c) Rites, R. E.; Willard, D. M.; Levinger, N. J. Phys. Chem. B 1998, 102, 2705 .

(8) (a) Bortulos, P.; Monti, S. Adv. Photochem. 1995, 21, 1. (b) Ramamurthy, V.; Eaton, D. F. Acc. Chem. Res. 1988, 21, 300. 6904.

(9) Hansen, J. E.; Pines, E.; Fleming, G. R. J. Phys. Chem. 1992, 96,

(10) Park, H.-J.; Mayer, B.; Woltschann, P.; Kohler, G. J. Phys. Chem. 1994, 98, 6158

(11) Agbaria, R. A.; Uzank, B.; Gill, P. J. Phys. Chem. 1989, 93, 3855

(12) (a) Sujatha, J.; Mishra, A. K. Langmuir 1998, 14, 2256. (b) Il'ichev, Yu. V.; Demyashkevich, A. B.; Kuzmin, M. G.; Lemmetiyen, H. J. Photochem. Photobiol. A 1993, 74, 51.

(13) Politi, M. J.; Brandt, O.; Fendler, J. H. J. Phys. Chem. 1985, 89, 2345 .

(14) Guha Ray, J.; Sengupta, P. K. Chem. Phys. Lett. 1994, 230, 75.

(15) (a) Chen, Y.; Rich, R. L.; Gai, F.; Petrich. J. W. J. Phys. Chem. 1993, 97, 1770. (b) Chen, Y.; Gai, F.; Petrich. J. W. J. Phys. Chem. 1994, 98, 2203.

(16) (a) Ireland, J. F.; Wyatt, P. A. Prog. Phys. Org. Chem. 1976, 12, 131. (b) Shizuka, H. Acc. Chem. Res. 1985, 18, 141.

(17) Pines, E.; Fleming, G. R. J. Phys. Chem. 1991, 95, 10448.

(18) Webb, S. P.; Yeh, S. W.; Philips, L. A.; Tolbert, M. A.; Clark J. H. J. Am. Chem. Soc. 1984, 106, 7286.

(19) Lee, J.; Robinson, G. W.; Webb, S. P.; Philips, L. A.; Clark J. H. J. Am. Chem. Soc. 1986, 108, 6538.

(20) Lee, J.; Robinson, G. W.; Webb, S. P.; Philips, L. A.; Clark J. H. J. Am. Chem. Soc. 1990, 112, 1353.

(21) Robinson, G. W.; Thistlewaite, P. S.; Lee, J. J. Phys. Chem. 1986, 90, 4224.

(22) Shizuka, H.; Ogiwara, T.; Narita, A.; Sumitani, M.; Yoshihara, K. J. Phys. Chem. 1986, 90, 6708.

(23) Krishnan, R.; Lee, J.; Robinson, G. W. J. Phys. Chem. 1990, 94 6365.

(24) (a) Pines, E.; Huppert, D.; Agmon, N. J. Chem. Phys. 1988, 88, 5620. (b) Huppert, D.; Kolodney, E.; Gutman, M.; Nachliel, E. J. Am. Chem. Soc. 1982, 104, 6949. (c) Huppert, D.; Jayaraman, A.; Mains, R. A., Sr.; Rentzepis, P. M. J. Chem. Phys. 1984, 81, 5596.

(25) (a) Bohne, C.; Redmond, R. W.; Scaiano, J. C. in Photochemistry in Organized and Constrained Media; Ramamurthy, V., Ed.; VCH: New York, 1991; p 92. (b) Attwood, D.; Florence, A. T. In Surfactant Systems; Chapman and Hall: London, 1983; pp 74-77.

(26) (a) Ware, W. R. In Photochemistry in Organized and Constrained Media; Ramamurthy, V., Ed.; (VCH: New York, 1991; p 563. (b) Toptygin, D.; Svobodova, J.; Konopasek, I.; Brand, L. J. Chem. Phys. 1992, 96, 7919.

(27) (a) Phillies, G. D. J.; Yambert, J. E. Langmuir 1996, 14, 3431. (b) Berr, S. S.; Coleman, M. J.; Jones, R. R. M.; Johnson, J. S. J. Phys. Chem. 1986, 90, 6492. (c) Berr, S. S.; Caponetti, E.; Jones, R. R. M.; Johnson, J. S.; Magid, L. J. J. Phys. Chem. 1986, 90, 5766. (d) Berr, S. S. J. Phys. Chem. 1987, 91, 4760. (e) Paradies, H. H. J. Phys. Chem. 1980, 84, 599. 